Original Investigation

\title{
Improving Medical Device Regulation: The United States and Europe in Perspective
}

\author{
CORINNA SORENSON* \\ and MICHAEL DRUMMOND*,
}

${ }^{*}$ London School of Economics and Political Science; ${ }^{\dagger}$ University of York

Context: Recent debates and events have brought into question the effectiveness of existing regulatory frameworks for medical devices in the United States and Europe to ensure their performance, safety, and quality. This article provides a comparative analysis of medical device regulation in the two jurisdictions, explores current reforms to improve the existing systems, and discusses additional actions that should be considered to fully meet this aim. Medical device regulation must be improved to safeguard public health and ensure that high-quality and effective technologies reach patients.

Methods: We explored and analyzed medical device regulatory systems in the United States and Europe in accordance with the available gray and peerreviewed literature and legislative documents.

Findings: The two regulatory systems differ in their mandate and orientation, organization, pre- and postmarket evidence requirements, and transparency of process. Despite these differences, both jurisdictions face similar challenges for ensuring that only safe and effective devices reach the market, monitoring realworld use, and exchanging pertinent information on devices with key users such as clinicians and patients. To address these issues, reforms have recently been introduced or debated in the United States and Europe that are principally focused on strengthening regulatory processes, enhancing postmarket regulation through more robust surveillance systems, and improving the traceability and monitoring of devices. Some changes in premarket requirements for devices are being considered.

Conclusions: Although the current reforms address some of the outstanding challenges in device regulation, additional steps are needed to improve existing policy. We examine a number of actions to be considered, such as requiring

Address correspondence to: Corinna Sorenson, LSE Health, London School of Economics and Political Science, London, UK (email: c.sorenson@lse.ac.uk).

The Milbank Quarterly, Vol. 92, No. 1, 2014 (pp. 114-150)

(c) 2014 Milbank Memorial Fund. Published by Wiley Periodicals Inc. 
high-quality evidence of benefit for medium- and high-risk devices; moving toward greater centralization and coordination of regulatory approval in Europe; creating links between device identifier systems and existing data collection tools, such as electronic health records; and fostering increased and more effective use of registries to ensure safe postmarket use of new and existing devices.

Keywords: medical devices, regulation, health care reform, comparative studies.

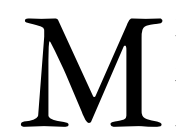

EDICAL DEVICES ARE SERVING AN INCREASINGLY CENTRAL role in clinical practice, improving patients' health and quality of life. The medical device industry and the areas of patient care it touches have grown considerably in recent years. For example, the annual revenues of the US medical device industry rose from approximately $\$ 85$ billion in 2001 to $\$ 146$ billion in $2009 .{ }^{1}$ While part of this growth is due to the greater use of medical devices already on the market, a large portion was driven by new market entrants. During the 2000s, more than 30,000 medical devices were cleared by the US Food and Drug Administration (FDA)'s 510(k) premarket notification pathway, and more than 300 new devices received original premarket authorization. ${ }^{2}$ Along with the higher number of new devices, these technologies have become more complex.

The growing number and sophistication of medical devices have introduced regulatory challenges. Recent debates and events in the United States and Europe have brought into question the effectiveness of the existing regulatory frameworks in both jurisdictions to ensure the performance, safety, and quality of new devices. In the United States, for example, the Institute of Medicine (IOM) recently called for the FDA to eliminate its $510(\mathrm{k})$ clearance process, maintaining that it was an unreliable screen for the safety and effectiveness of devices. ${ }^{3,4}$

Industry has generally taken a different stance, focusing on concerns that the US regulatory system is too slow, risk adverse, and expensive. The European system is therefore often viewed as superior, given its somewhat faster regulatory process for devices and earlier access to some high-risk technologies (eg, coronary stents, replacement joints). ${ }^{5,6}$ However, European regulators have also faced criticism. In a commentary in the British Medical Journal, Freemantle ${ }^{7}$ asserted that the current European regulatory framework for medical devices, through the Conformité européenne (CE) marketing process, is inadequate to 
provide sufficient safeguards for technologies that affect morbidity, mortality, and health-related quality of life. The cited inadequacies include inferior regulatory evidence standards, nontransparent decisionmaking processes, and insufficient postmarket surveillance to ensure devices' safety and long-term performance. The European Commission has echoed such concerns, stating a need to "adapt the European regulatory framework in order to secure patients' safety while favouring innovation." 8 Recent market recalls of articular surface replacement hip prostheses, Poly Implant Prothése (PIP) breast implants, and PleuraSeal for lung incisions, many of which were denied approval by the FDA, have further heightened concerns about current regulatory practices. ${ }^{9-11}$

Given that the United States and Europe have recently introduced or are currently debating reforms of medical device regulation, it is an opportune time to examine the current regulatory policies and practices in both jurisdictions and identify areas for additional improvement. Despite the recent studies comparing medical device regulation in the United States and Europe, ${ }^{12,13}$ there is little in-depth discussion of the key issues in reforming the existing regulatory frameworks and strategies to be considered and employed to improve medical device regulation. The purpose of this article is to fill this gap. First, we offer a brief comparative overview of medical device regulation in the United States and Europe. Second, we examine the main challenges facing the regulation of devices, followed by an analysis of recent and ongoing reforms. We close with a discussion of additional policies and practices that could be considered in current reform plans, or in the future, to strengthen the regulation of medical devices in both jurisdictions.

\section{Comparative Overview of US and European Medical Device Regulation}

\section{United States}

The 1976 Medical Device Amendments gave the FDA the primary authority to regulate medical devices and to substantiate "reasonable assurance of safety and effectiveness" before allowing manufacturers to market their products. ${ }^{14}$ This legislation has subsequently been updated with the Medical Device User Fee and Modernization Act (MDUFMA) of 2002, which established sponsor user fees for application reviews and set certain performance goals for the agency. 
The FDA assigns devices to one of three regulatory classes based on their intended use, whether the device is invasive or implantable, and the risk posed by the device to the user. As Table 1 shows, the device class determines the level of evidence and evaluation required to demonstrate safety and effectiveness. Low-risk Class I devices are generally exempt from premarket notification $(510[\mathrm{k}])$ and FDA clearance before being marketed, although their manufacturers are subject to general controls, such as registering their name and products with the FDA. Medium-risk Class II devices usually are required to clear the $510(\mathrm{k})$ review process, which determines principally whether the new device is substantially equivalent to a legally marketed (predicate) device. Substantial equivalence means that the device performs in a manner similar to that of the predicate in its intended use, technological characteristics, and safety and effectiveness. ${ }^{15}$ If a device is determined to be substantially equivalent, a clinical trial is usually not required to prove its safety or effectiveness. Other requirements (special controls) may be imposed, however, such as those for labeling requirements and postmarket surveillance. ${ }^{12}$ If the FDA deems a device to not be substantially equivalent, the manufacturer can petition for reclassification or file a de novo application.

High-risk Class III devices require closer scrutiny. These technologies are generally required to undergo the most formal review process for devices: premarket authorization (PMA), in which a device must demonstrate safety and effectiveness through the submission of clinical studies. Devices in this class that have been created from changes to previously PMA-approved devices may not be required to generate additional clinical evidence. ${ }^{16,17}$ Novel devices without a predicate are automatically classified as Class III, regardless of their risk profile. But if the device is classified as low to moderate risk, the manufacturer can apply for reclassification to Class II or I through the de novo process and need not undergo PMA, a process we discuss further in subsequent sections.

To safeguard public health once a device is on the market, the FDA requires a range of postmarket surveillance activities (Table 1), including adverse event reporting by manufacturers and user facilities (via the Medical Device Reporting [MDR] program) and postmarket studies to ascertain and monitor the device's safety and effectiveness. ${ }^{12}$ The agency also supports a number of surveillance data networks, such as MedWatch, the Medical Device Surveillance Network (MedSun), and the Medical Device Epidemiology Network Initiative (MDEpiNET), to identify and 


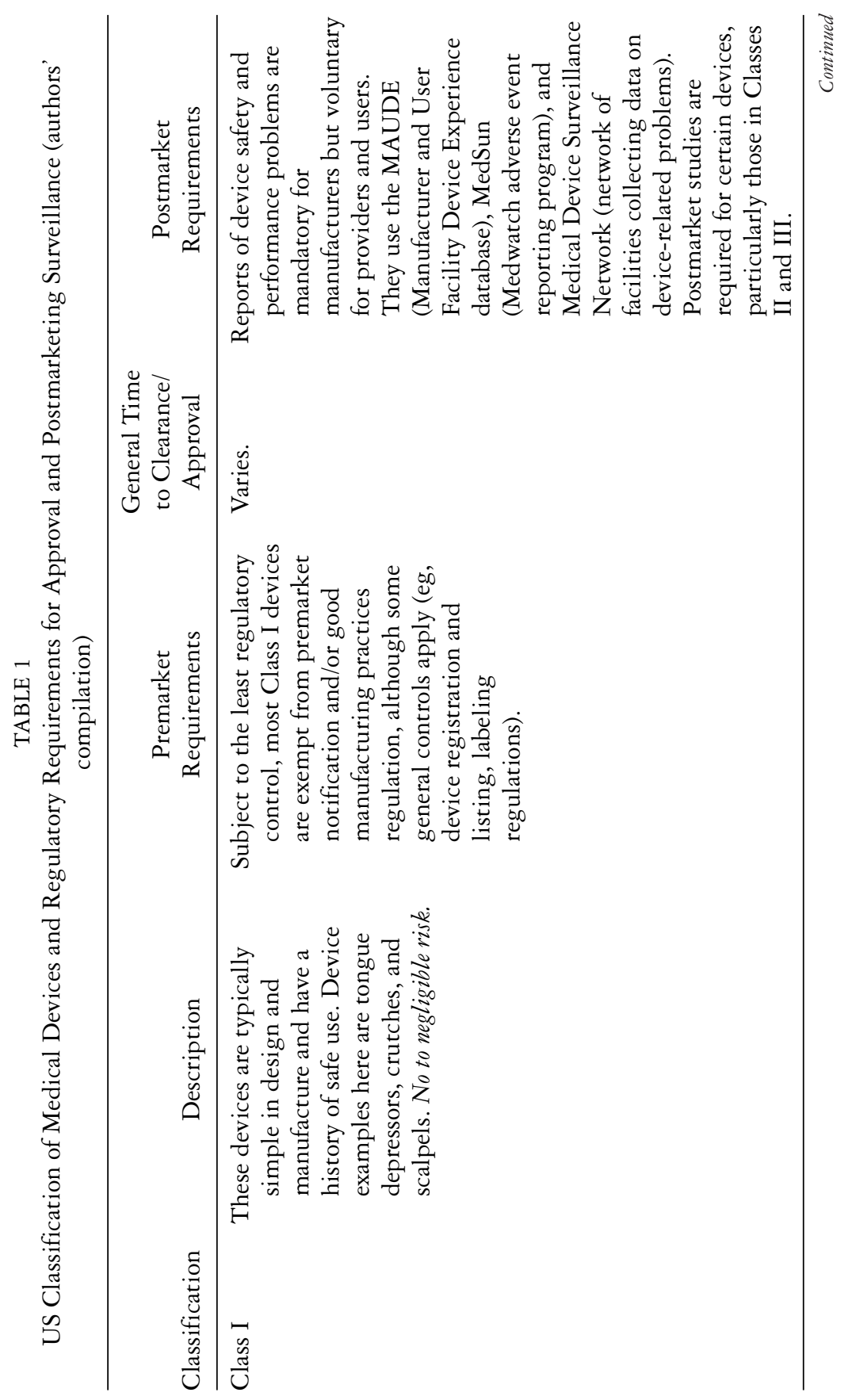




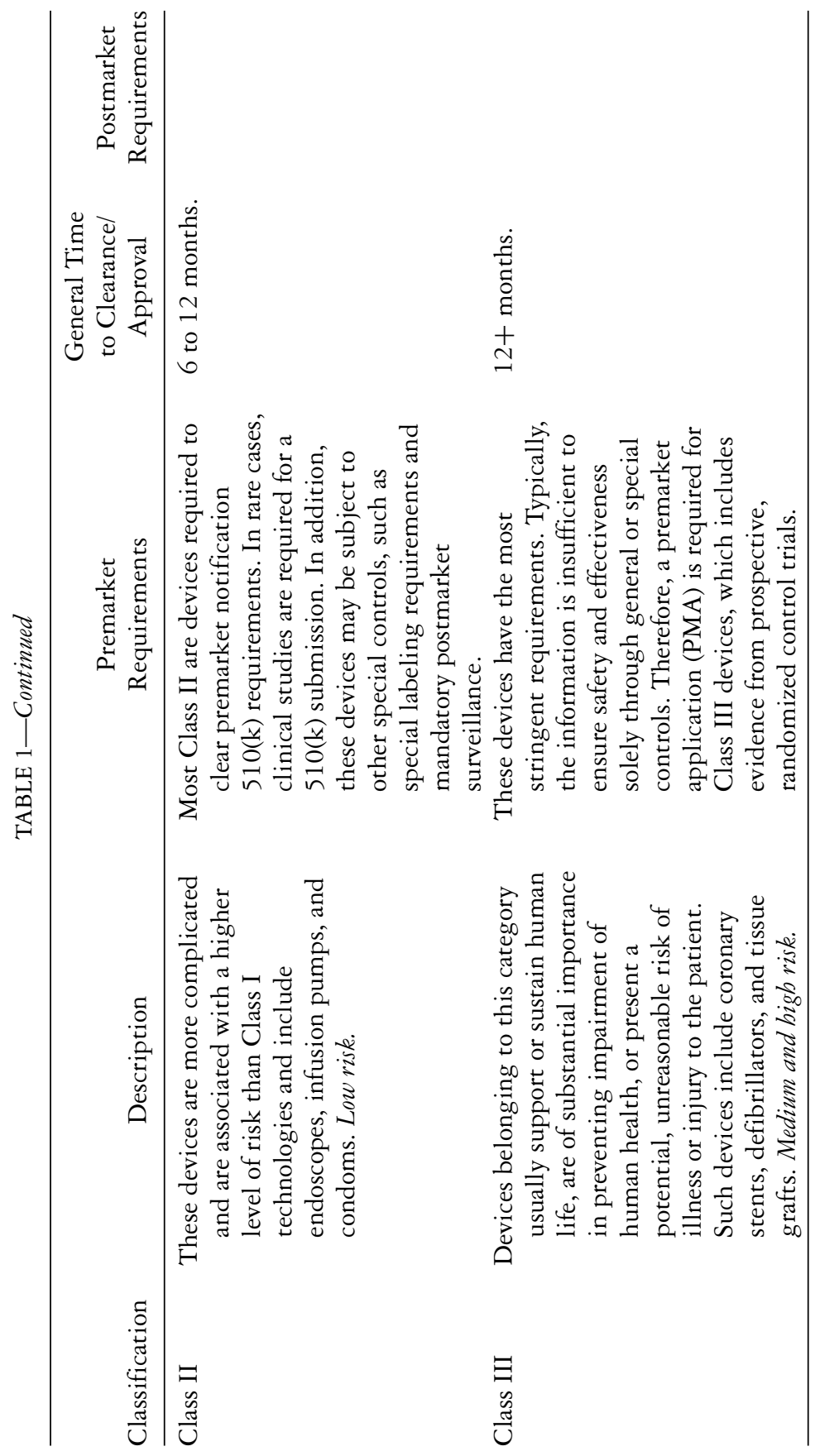


address safety problems and advance epidemiological methods for device surveillance.

\section{Europe}

Until the 1990s, each member state had its own approach to regulating devices. To regulate a diverse and complex market and promote the "internal market" in Europe, new regulations, known as the New Approach Directives, were introduced by the European Council that defined the "Essential Requirements" to ensure devices' safety and performance. ${ }^{12}$ These requirements apply to all countries. Therefore, if a device meets the requirements and receives a CE mark in one country, it can be marketed in all member states. A CE mark certifies that a device is safe and functions according to the intended purpose described by the manufacturer. Under these directives, devices are categorized into four classes according to the degree of risk associated with their intended use (Table 2).

Similar to those of the United States, Europe's evidence requirements for market authorization increase with the degree of risk associated with the device. Manufacturers of low-risk devices (Class I) are required only to self-declare conformity with the Essential Requirements to a national "Competent Authority," such as the Medicines and Healthcare Products Regulatory Agency (MHRA) in the United Kingdom. More moderate- and high-risk devices (Classes IIa, IIb, and III) require a combination of clinical and nonclinical data on the device being evaluated. If available, data for an equivalent device already on the market may be submitted. Although clinical studies are generally requested for high-risk Class III devices, the evidence requirements are vague, not available to the public, and nonbinding for manufacturers and studies need not be randomized. ${ }^{18}$ For manufacturers claiming similarity to an existing product, a comparative literature review typically suffices.

Manufacturers of these devices select and pay one of about 80 forprofit, private "Notified Bodies" to evaluate their device and receive a CE mark. Award of a CE mark is based on an evaluation of safety and performance (that a device functions as intended) and not effectiveness (clinical benefit).

Once a device is on the market, manufacturers are required to report all serious adverse events to the Competent Authorities. In Europe, this 


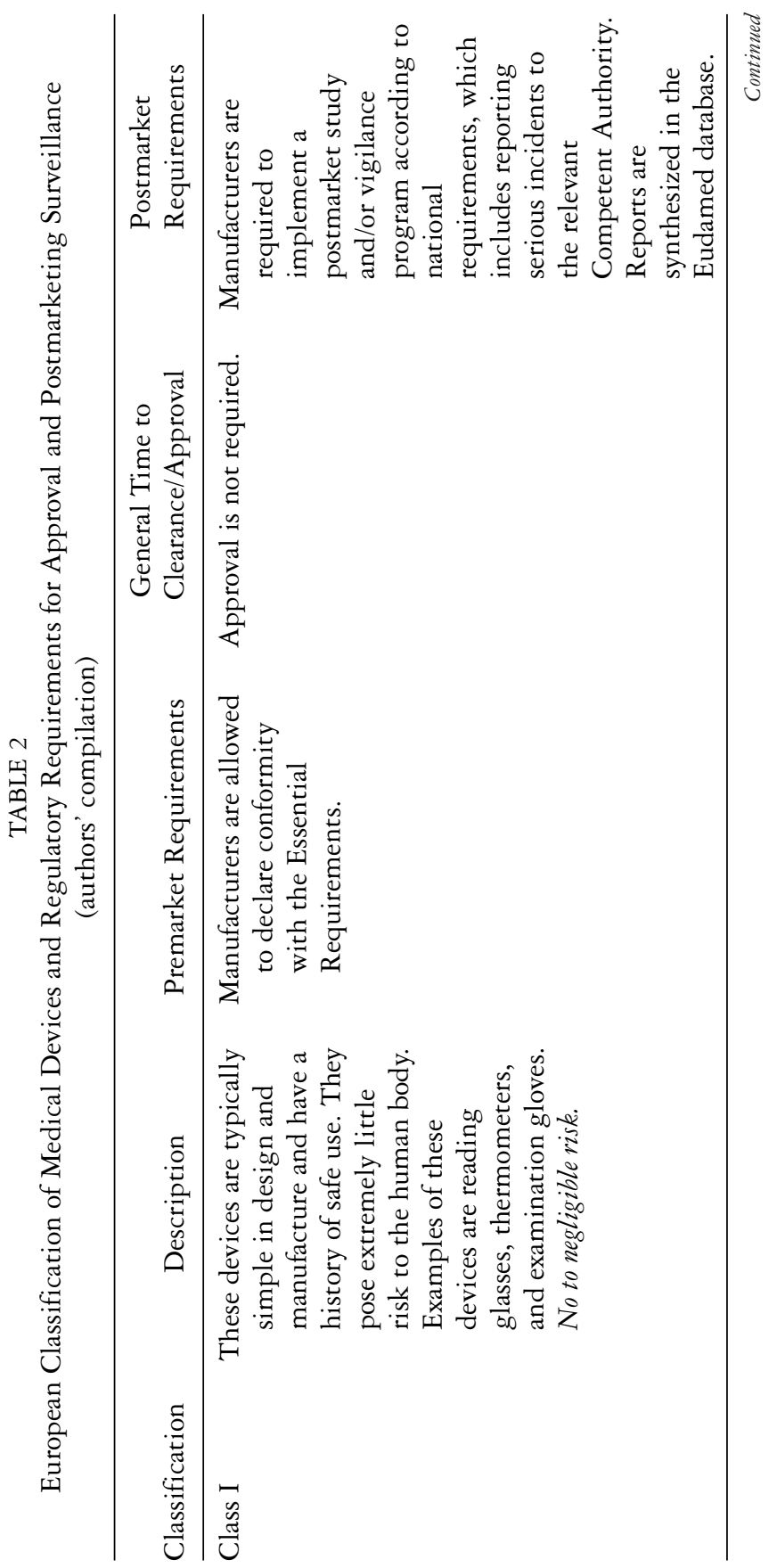




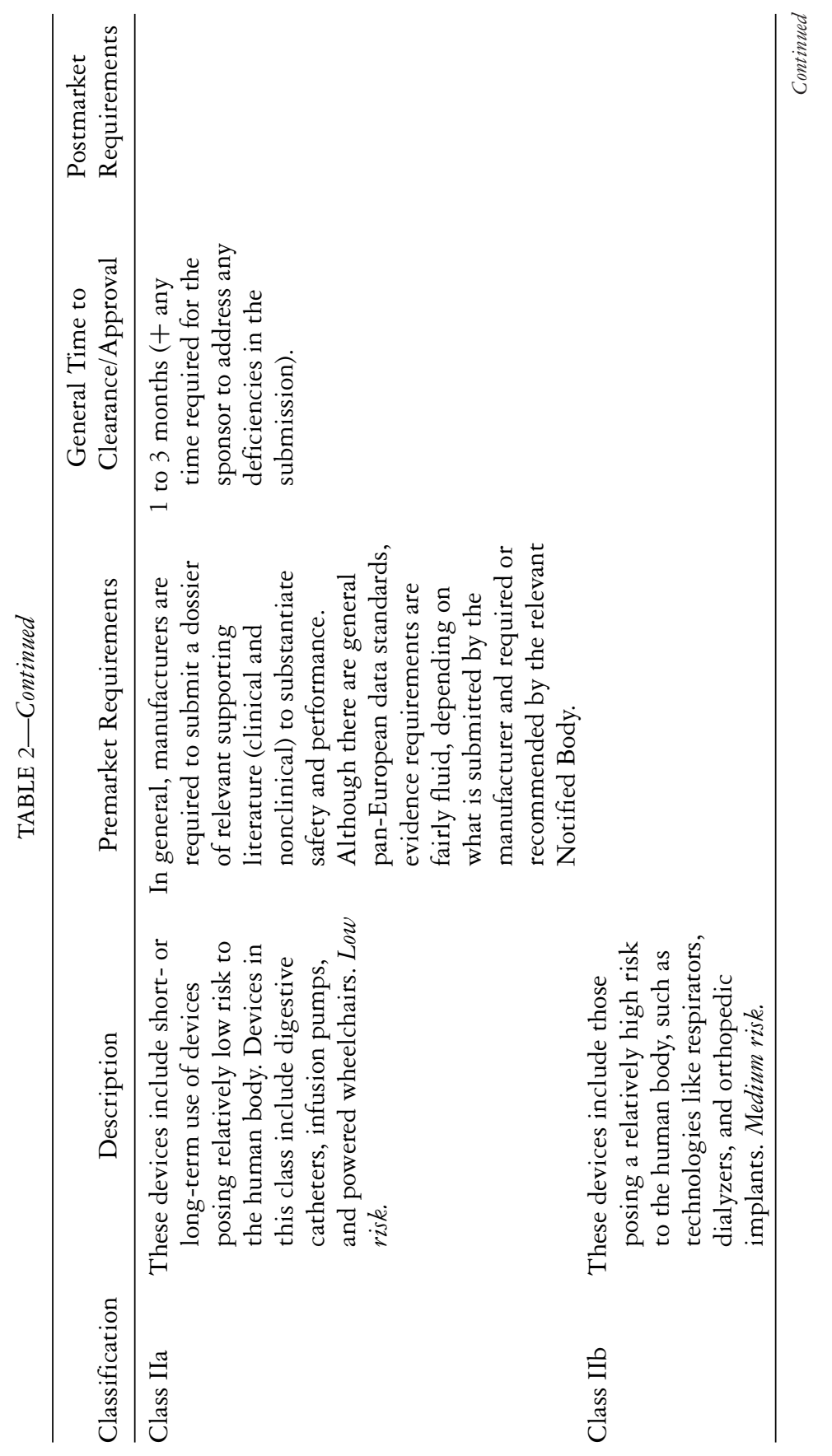




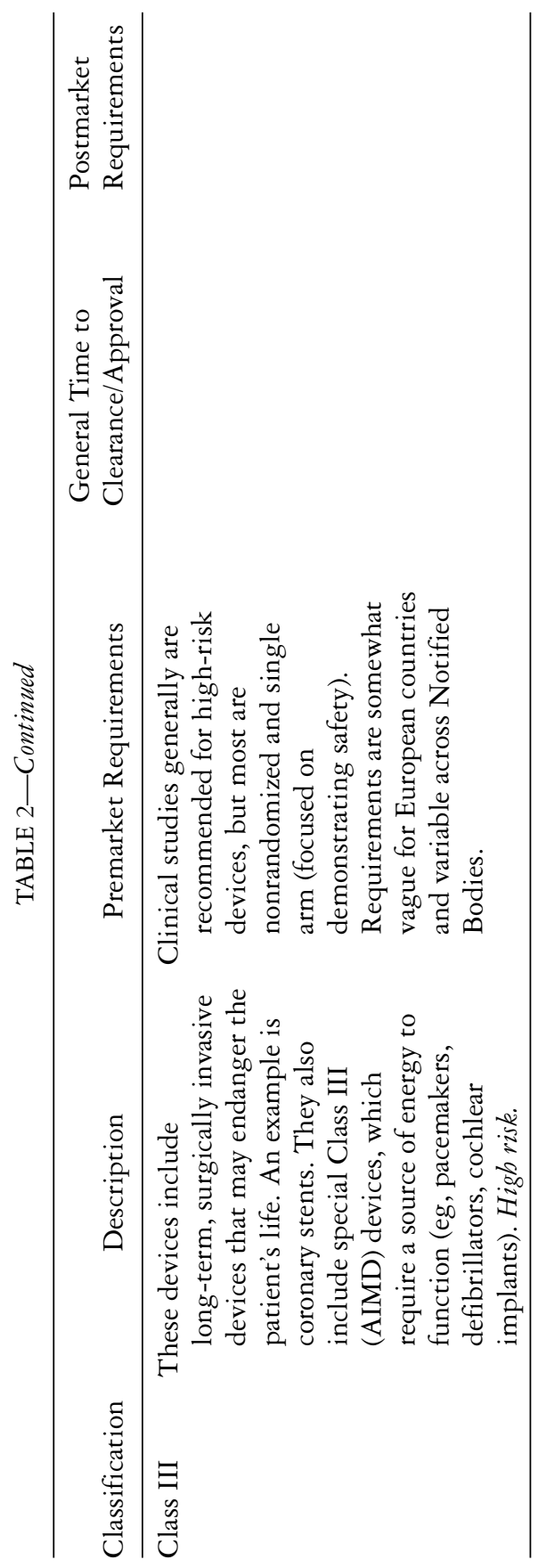


information is collated into a central database, the European Databank on Medical Devices (Eudamed). In addition to vigilance information, Eudamed contains data on manufacturers; certificates issued, modified, suspended, withdrawn, or refused; and clinical investigations. The use of Eudamed has been mandatory since 2011. Postmarket studies also may be required if a device's medium- or long-term safety and performance are not known from previous use of the device or when other postmarket surveillance activities would provide insufficient data to address risks.

\section{Comparing the United States and Europe}

The US and European approaches to medical device regulation have fundamental differences. For example, the FDA was established to promote and protect public health through the regulation of medical products, whereas the European system of Notified Bodies developed as part of a broader initiative to strengthen innovation and industrial policy across Europe. Notified Bodies therefore were not designed to function as public health agencies. Instead, the protection of public health lies largely with the Competent Authorities, with the extent of their role varying widely among member states. Kramer and colleagues ${ }^{12}$ believe that these differences help explain why the United States and Europe have adopted different regulatory processes and evidence requirements for devices. For instance, in Europe devices must prove only that they work as intended, whereas in the United States devices require evidence of effectiveness.

Another key difference relates to the organization of the regulatory systems. In the United States, the FDA oversees all regulation of devices. In contrast, the European system confers significant authority on a collection of governmental (Competent Authorities) and private (Notified Bodies) bodies to oversee device evaluation, market approval, and postmarket surveillance. The US approach theoretically allows for better coordination and ease of enforcing regulatory requirements, although as mentioned earlier, some commentators believe that greater centralization results in a rigid, lengthy, and costly regulatory process. ${ }^{5,6}$ While the more flexible European approach may grant faster market access to certain devices, it is not without problems. For example, evidence standards have been found to differ across Notified Bodies, ${ }^{19}$ which may encourage manufacturers to seek a CE mark from a less rigorous body. Decentralization also hinders the collection and analysis of safety data, especially for rare, but life-threatening, adverse events, for which a 
substantial amount of patient information is required to detect potential problems. $^{20}$

\section{Outstanding Challenges in US and European Medical Device Regulation}

Despite the differences between the US and European systems, both jurisdictions face similar outstanding challenges to effective medical device regulation. Next we discuss several issues needing improvement.

\section{Establishing and Upholding Appropriate Evidence Requirements}

Requiring sufficient evidence (and applying rigorous review mechanisms) to safeguard public health and certify effectiveness, especially for high-risk devices, is perhaps the greatest challenge currently facing both US and European device regulation. In the United States, there are concerns that too many high-risk devices are evaluated through less rigorous review mechanisms. ${ }^{3}$ Over the last 10 years, only about $2 \%$ of medical devices have undergone PMA. ${ }^{21}$ A GAO study ${ }^{22}$ found that between 2003 and 2007, only 79\% of Class III devices actually underwent PMA, with the remainder proceeding through the $510(\mathrm{k})$ pathway. Unlike PMA, direct evidence of safety and effectiveness is usually not required for $510(\mathrm{k})$ submissions, and only $10 \%$ to $15 \%$ of submissions contain any clinical data. ${ }^{23}$ Furthermore, devices deemed substantially equivalent to devices previously cleared by the FDA do not need to go through the premarket approval process, even if that previous model was never assessed for safety and effectiveness or recalled for a major safety defect. ${ }^{24}$ One study investigating a cohort of high-risk recalls in the United States showed that $71 \%$ of such devices had previously been cleared through the $510(\mathrm{k})$ process and another $7 \%$ had been exempt from review. ${ }^{25}$ Besides the quantity of robust evidence are quality issues. Based on an internal analysis by the FDA, more than half of the $510(\mathrm{k})$ submissions it received have quality problems, including incompleteness or failure to address basic elements such as a description of the device and proposed indications for its use. ${ }^{26}$

Even the PMA has challenges. FDA mandates only that PMA applications provide a reasonable assurance of safety and effectiveness. ${ }^{27}$ The 
evidence available suggests that this typically means applications were approved based on a single clinical study. ${ }^{28}$ In addition, only a minority of trials are randomized or blinded, use an active control group and hard end points, and are consistent in the way they account for patients and report data. ${ }^{28-30}$ Such standards differ for drugs, which are expected to show "substantial evidence [of safety and effectiveness]" and for which uncontrolled or partially controlled studies are not considered sufficient for approval. ${ }^{31}$ An alternative perspective (often taken by the FDA, industry, and some analysts) is that devices are different from drugs and therefore should not be held to the same standards. ${ }^{32}$ In particular, devices introduce challenges that render clinical trials less feasible. For example, for a surgical device, it is difficult to randomize patients for surgery or no surgery and/or blind patients or physicians. Moreover, the many different types of devices make it difficult to apply one evidence standard to all devices.

Another issue arises from a stipulation of the Medical Device Amendments of 1976, which established varying safety standards for devices that the FDA deems as low, medium, and high risk, as previously discussed. The law applied immediately to new types of devices and directed the FDA to retroactively classify products that were already on the market when the law passed. This meant that Class I and II devices underwent review for substantial equivalence to devices already on the market. But even though Class III devices were intended for PMA, they were allowed to receive review for substantial equivalence temporarily until the FDA down-classified them or required PMA.

Congress always intended Class III devices to undergo PMA, and in 1990 under the Safe Medical Devices Act, it directed the FDA to establish a time line to complete the transition to PMAs for all devices that were to remain in Class III. ${ }^{3}$ The FDA, however, still has not classified some of the "grandfathered" devices. As of early 2013, 19 different types of Class III devices are allowed to reach patients through $510(\mathrm{k})$ clearance. ${ }^{24}$ Consequently, potentially high-risk devices continue to reach the market without ever being tested in humans. One such example is metal-on-metal hip implants. Ardaugh and colleagues ${ }^{24}$ traced the 510(k) history of the DePuy ASR XL Acetabular Cup System and found that in most cases, the predicates used for clearance were not metal-on-metal and were substantially different in design from the ASR XL or their clinical performance was poor. Almost a year after its approval, the ASR's high revision rate was discovered when it was 
compared with all other total conventional hip prostheses in the Australian Joint Registry. By this time, ASRs had been implanted in millions of patients, many of whom suffered serious harm and, as a result, needed additional procedures to replace the device. ${ }^{10}$

In Europe, the majority of Class III devices need only to demonstrate their safety and performance, not that they directly benefit patients, and there are no requirements to verify the adequacy of submitted clinical data. In most cases, the submission of robust clinical data is limited, and often the evidence submitted is from laboratory testing, literature reviews, or small clinical trials. ${ }^{11}$

A less stringent premarket review process increases the risk that later studies may demonstrate that the device has no benefits or identify important adverse events that did not emerge at the time of market authorization. For example, although 10 times more drug-eluting stents are approved in Europe than in the United States, many of those approved offer no advantage over other treatment alternatives for preventing restenosis or have worse outcomes than other stents. ${ }^{33}$ Other devices approved in Europe have been withdrawn from the market after later studies demonstrated poor performance or unexpected complications. ${ }^{19}$ The Notified Bodies' lack of uniform evidence requirements is another, related concern. Such diversity results in regulatory unpredictability as well as inconsistent evidence standards being applied to similar devices.

Overall, US and European evidence requirements for devices introduce not only risks to patients but also the wrong incentives to generate the needed evidence to better understand and evaluate the benefits and risks of new devices. Considering that manufacturers often take advantage of existing evidence from already marketed devices to gain approval for a new device, they are reluctant to undertake new clinical studies. In addition, because later devices may be able to claim equivalence, the first manufacturer to market does not have a very strong incentive to undertake extensive clinical studies. This may be exacerbated by the fact that many device manufacturers are small-to-medium enterprises (SMEs) that often lack the requisite expertise and resources to conduct large clinical studies. Taken together, all these issues suggest that when a device (or procedure using a device) enters clinical practice, the information about its efficacy and short- and long-term safety is meager. Accordingly, the adoption of a new device may be driven more by marketing and the enthusiasm of clinicians than by evidence. 


\section{Monitoring and Evaluating Postmarket Device Safety and Effectiveness}

Despite the various mechanisms to collect postmarket surveillance data in the United States, such as the MedWatch and MedSun systems, the reporting of adverse events remains weak. Although by law, manufacturers must report deaths or serious adverse events, they are not required to if they decide that the events are unrelated to the device. ${ }^{34}$ Furthermore, voluntary reporting by providers, patients, and health facilities is somewhat rare and may be subject to reporting biases. A 2009 government report ${ }^{35}$ pointed out that only $6 \%$ of adverse event reports come from health care providers and users. Several factors contribute to underreporting, including the reports' voluntary nature, fear of litigation, difficulties in connecting problems with a device, and failure by patients and providers to understand their obligation to report. ${ }^{36}$ Moreover, clinicians may not have sufficient time or support to collect and submit data routinely. The FDA's ability to detect potentially unsafe devices is further hampered by the fact that many postapproval studies required as a condition of approval are actually not conducted or are of such poor quality as to not produce meaningful postmarket evidence. ${ }^{34}$

European postmarket systems face similar challenges. Manufacturers are required to report adverse events to Competent Authorities, but the events' inclusion in Eudamed is dependent on the Competent Authorities, who are not mandated to report. Only a few national Competent Authorities provide the majority of adverse event reports and public notifications of device-related safety concerns, ${ }^{12}$ and no mechanism is available for providers and patients to report adverse events. Eudamed allows information to be exchanged only between national Competent Authorities and the European Commission, and it is not available to the public. In addition, Kramer and colleagues ${ }^{12}$ noted that the coordination and analysis of postmarketing reports from Eudamed are highly variable. Consequently, to date, Eudamed has had limited utility. While guidelines have been issued to address some of these issues, they are vague and remain at the discretion of manufacturers. Poor adverse event reporting, in addition to fraud and poor postmarket regulatory oversight, was associated with the recent PIP implant recall. ${ }^{\text {? }}$

Without systematic postmarket data collection, it is difficult for clinicians, other health professionals, and regulatory agencies to understand to whom health care is provided and the actual outcomes of particular procedures or the use of devices once they are on the market. This is 
particularly important in the case of medical devices, for which evidence regarding their performance is frequently limited when they are first used. Moreover, often only through the actual use of a device are unforeseen problems related to safety and performance identified and addressed. ${ }^{37}$ For example, an analysis of stent implantation between 2003 and 2004 using the Swedish Coronary Angiography and Angioplasty Registry (SCAAR) found that patients treated with drug-eluting stents (DES) had a higher rate of mortality than did those receiving bare-metal stents. ${ }^{38}$ The findings caused upheaval and prompted an immediate decline in the use of DES and an urgent review of their safety. A follow-up SCARR study (with data extended to 2010), however, found that the new generation of DES was associated with lower rates of restenosis, stent thrombosis, and mortality. ${ }^{39}$ The difference in outcomes was largely explained by cardiologists' improved practice, with greater use of the device and the introduction of better stents.

\section{Ensuring Adequate and Transparent Information Exchange on the Benefits and Risks of Devices}

The public's demand for accessible and transparent information about devices and the regulatory process has grown in recent years, and both the United States and Europe have taken action to improve the exchange of information with stakeholders. For instance, the FDA produces publicly available information about its regulatory pathways for various device types and associated evidence requirements; publishes advisory committee input on new devices; and summarizes its justification for its approval of high-risk devices and information about associated adverse events. The agency also requires the disclosure of any financial interests that a clinical investigator may have in a device or product sponsor. Although the FDA does not publicly disclose this information, in its recent guidance on financial disclosure, the agency noted that it intends to provide information about the number of clinical investigators as well as financial information in the product reviews it posts for an approval decision. ${ }^{40}$ In Europe, collected postmarket data are shared with Competent Authorities, and individual Competent Authorities provide on their websites information regarding their operations.

Achieving an open and accessible information exchange still is elusive. In the United States, much of a sponsor's application for a new device 
remains proprietary, as is information about applications not approved. Moreover, European Notified Bodies have no obligation to publish their decision-making process, the evidence provided by sponsors, or the basis for granting a CE mark. And postmarket data are not shared with the public.

\section{Current Reforms to Improve Medical Device Regulation}

The current regulatory systems for medical devices clearly must be improved. Next we discuss several areas of reform that are under way or have been proposed.

\section{Enbancing Existing Regulatory Frameworks}

The growing number and complexity of medical devices are challenging the current regulatory frameworks. To address some of these challenges and those associated with the FDA's device review programs in general, in mid-2012 the United States passed the Food and Drug Administration Safety and Innovation Act (Public Law 112-144). ${ }^{41}$ Among its various provisions, the law supports enhanced transparency and justification of significant agency decisions regarding device applications; a change in the agency's guidance when device modifications require premarket notification before marketing; programs to improve the device recall system; modifications of the de novo application process; new procedures to reclassify devices previously grandfathered into the system; and mechanisms to enhance postmarket surveillance, such as the inclusion of devices in the Sentinel surveillance system. While some of these actions are intended to make the regulatory process more efficient, such as changes to the de novo application process, others (eg, device reclassification, Sentinel) strive to better safeguard public health.

Since the publication of the IOM report, the FDA has introduced other measures to improve the existing $510(\mathrm{k})$ process, although it did not adopt the IOM's overall recommendation to eliminate the program altogether. The FDA's initiatives include new guidances to improve the program's predictability and effectiveness (eg, guidance to improve the quality and performance of clinical trials and clarify when changes in a device warrant a new $510[\mathrm{k}]$ submission); additional programs 
to fortify the $510(\mathrm{k})$ systems, including analyzing the use of multiple predicates; and training for agency staff and industry on various facets of the program.

The European Parliament is currently considering proposals to reform the EU's legislation on medical devices and in vitro diagnostics put forward by the European Commission and the parliament's Rapporteur and Committee on the Environment, Public Health and Food Safety. ${ }^{42,43}$ The commission's proposal offers insubstantial modifications to European device regulation. The parliament rapporteur and committee, however, have called for far more oversight and transparency than the current system offers, with extra scrutiny of the highest-risk devices, including a more centralized premarket authorization process. Industry groups are fiercely debating the proposals, particularly the parliament rapporteur's and committee's measures, claiming that they would slow patients' access to beneficial technologies and hamper the "edge that industry has here in Europe." ${ }^{4}$

The latest parliament vote on the reforms sidestepped a centralized premarketing authorization system but supports a number of measures clarifying the roles and responsibilities of the involved parties (eg, national authorities, clinical experts), fostering coordination and harmonization across member states, enhancing the oversight and standards associated with Notified Bodies, and increasing the transparency and traceability of devices. Among other things, this means that Notified Bodies will continue to grant market approval through CE certification but will face increased oversight and quality assurance by the Competent Authorities and a new Medical Device Coordination Group (MDCG), especially for high-risk devices. The MDCG, composed of experts and representatives of relevant stakeholder groups, is intended to provide advice to the European Commission and to assist the commission and Competent Authorities in ensuring the harmonized implementation of the reforms. For instance, before a Notified Body can issue a certificate, the MDCG will have the ability to request a preliminary conformity assessment on which it can issue comments within a deadline of 60 days. A small group of independent scientific experts will support the MDCG in its decision making.

Although the Notified Bodies will retain much of their current authority, the new legislation does result in greater regulatory centralization. The European Commission will be more involved in the review and approval of devices. For example, certain members of the commission, 
along with the MDCG and other experts, will advise on the designation of Notified Bodies and ensure that the member states charge comparable fees. The commission also will be responsible for maintaining Eudamed, which is central to the implementation of some of the new rules, particularly with regard to enhancing devices' transparency and traceability.

Other significant changes are requirements that certain devices (eg, high-risk implantables) undergo assessment by specialized Notified Bodies designated by the European Medicines Agency (EMA), the regulator for pharmaceuticals. Notified Bodies will be expected to have permanent in-house competent personnel and technical and medical expertise related to devices and will be subject to assessments of compliance and ongoing monitoring. Manufacturers also will be subject to unannounced inspections, and those that commit fraud will face serious penalties such as imprisonment.

\section{Strengthening Premarket Evidence Standards and Requirements}

The impact of the US reforms on device evidence standards and requirements is somewhat limited, with the most significant developments being changes to the de novo application process and the reclassification procedures.

In the past, the de novo process required manufacturers to submit a $510(\mathrm{k})$ application, which is exhaustively reviewed by the FDA before a device can receive a "not substantially equivalent" determination. If deemed not equivalent, the device will automatically receive a Class III designation. Only then can the manufacturer submit a de novo request to have the device reclassified from a Class III to a Class II or I designation. This complicated and somewhat cumbersome two-step process has resulted in the rare use of the de novo route and in unnecessary delays when it has been used. For example, only 54 de novo classifications have been made since the process went into effect in 1998, and once a de novo application is submitted, it takes the FDA an average of 240 days to review it. ${ }^{45}$ Ladin and Imhoff ${ }^{45}$ found that de novo review times have increased in recent years and are sometimes longer than PMA reviews. The main reasons for the few de novo applications and the rise in review times are unclear. But procedural inefficiencies are likely a cause, as well as the more complex devices being reviewed, the greater use of multiple predicates, ${ }^{3}$ and the poor quality of applications. ${ }^{46}$ Consequently, some 
innovative, lower-risk technologies may have been inappropriately subjected to PMA approval or delayed market entry because of lengthy de novo review times.

The new de novo process outlined in recent reforms simply requires that manufacturers submit a request to the FDA for de novo classification, which will streamline the procedure by removing the requirement for $510(\mathrm{k})$ application and review. The FDA will have 120 days to issue a decision on classification. A recent analysis by the agency suggests that since 2011 , the average $510(\mathrm{k})$ review time has decreased, ${ }^{47}$ and the new process should raise the number of de novo applications and further shorten review times.

The reforms also make it easier for the FDA to reclassify "grandfathered" devices as either Class I or Class II or to call for a PMA application. The main change is that the FDA will no longer be required to issue a reclassification regulation in order to reclassify a device, which used to require an economic review of the potential impact of reclassification. This process can take years to complete. As a result of the reform, the FDA can accomplish the same thing by administrative order, which should expedite the process. To date, 6 types of devices have been proposed for reclassification, including metal-on-metal hip implants, which are required to meet PMA review. ${ }^{10}$ One area of uncertainty with the new process is that the reform called for all reclassifications to go before a panel. Consequently, it may now take longer for the FDA to downclassify certain devices, and additional time may be needed to assemble the requested panels.

The European reforms generally uphold the safety and performance requirements outlined in the Essential Requirements under the current approach, even in the case of high-risk devices. ${ }^{42}$ But the reforms do require greater harmonization of evidence standards across Europe, ${ }^{42}$ and it is encouraging that the latest reform language suggests that the "clinical evaluation" of devices may include not only safety and performance but also clinical benefits. ${ }^{43}$

\section{Improving Monitoring of Postmarket Patient Safety and Quality of Care}

In the United States and Europe, reforms have focused largely on improving postmarket regulation to better safeguard patients' safety and quality of care. Both jurisdictions introduced a unique device identifier 
(UDI) requirement to enhance the traceability of devices. In the United States, device manufacturers will be required to place a UDI on the device's label. Some devices will also need to be directly marked with the UDI itself. In addition, accompanying device information will be made available through the Global UDI Database (GUDID). As the FDA explained, the purpose of the UDI system is to provide speedy identification of devices associated with adverse events, assist with faster and more efficient resolution of device recalls, and deliver an easily accessible source of definitive device identification.

The UDI system will allow devices to be incorporated into the Sentinel Initiative. Sentinel proactively monitors various data sources rather than relying on spontaneous reporting from manufacturers and health care providers, which will enable the more timely identification of safety issues. The system, however, was initially designed to track drugs (via a National Drug Code), and adapting Sentinel to monitor devices has been difficult because of problems with identifying specific devices in the available data. The UDI system will help address this issue by allowing information about specific devices to be integrated into electronic patient health records and health insurance claims, two of Sentinel's main data sources. UDI also will be able to improve other types of postmarket surveillance, such as registries, and provide important information to and from relevant stakeholders as devices move from the manufacturer to the health system and eventually become part of patient care. With certain exceptions, implementation of these requirements will be based on device class (first applied to implantable, life-saving, or life-sustaining devices) over a period of 5 years from the Final Rule, which was recently released.

The goals and general requirements of the European UDI system are similar to those of the United States to ensure a harmonized approach to device traceability and a globally accepted UDI system. The European approach will also have a Europe-wide UDI database. Most likely, the UDI information will be included in Eudamed. ${ }^{43}$ It remains to be seen whether member states will decide to develop their own UDI systems, which could reduce the UDI's usefulness, but that seems unlikely if it becomes part of Eudamed.

In addition to the UDI system, the US reforms aim to improve the device recall system. In particular, the FDA is encouraged to proactively identify strategies for using recall information to improve the safety of devices and create tools to identify frequently recalled devices and 
the common root causes of safety problems. In addition, to ensure and speed up the completion of postmarket studies, the FDA now requires manufacturers to submit study plans within 30 days of the agency's order and to initiate studies within 15 months.

Similarly in Europe, the reform proposals under discussion are considering several actions to achieve a more robust postmarket surveillance system. The role of Eudamed will be expanded. Member states will be required to submit information about the registration of manufacturers and devices; any CE certificates issued, modified, withdrawn or rejected; vigilance activities and outcomes; and any clinical investigations. Manufacturers of high-risk devices will also have to submit a written report of the device's safety and performance and the outcome of the clinical evaluation, with the expectation that the summary be updated annually. The reform language also states that Eudamed should be robust and transparent and ensure access by the public and health care professionals to key parts of the database, such as vigilance and market surveillance information. ${ }^{43}$ In addition, member states will use compatible reporting forms for adverse events and device deficiencies, and time lines for reporting will be established according to the severity of the event reported.

\section{Additional Directions for High-Performing Medical Device Regulation}

While the current reforms in the United States and Europe will go some way to address the current weaknesses in both systems, additional actions could be taken to further improve medical device regulation (Tables 3 and 4).

\section{Premarket Evidence Requirements}

In Europe, there is no agreed-on requirement that the approval of medium- and high-risk devices be based on high-quality evidence of benefits that are relevant to patients. Patient safety could be improved by requiring an assessment of short- and long-term benefits and harms in well-designed RCTs, with the use of blinding and hard end points whenever possible. These requirements should be the same for all member states (and Notified Bodies). There should be no region or body of 


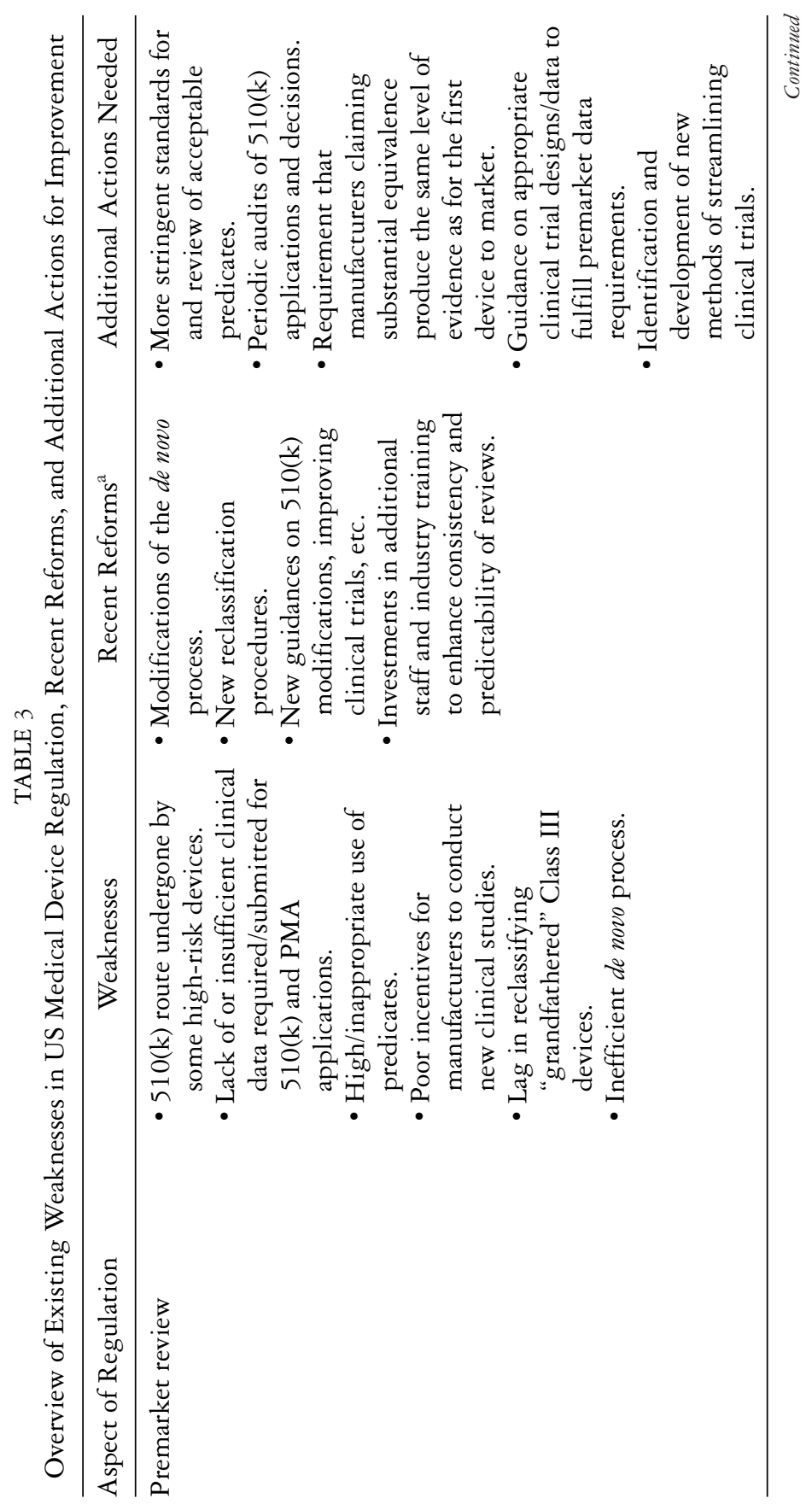




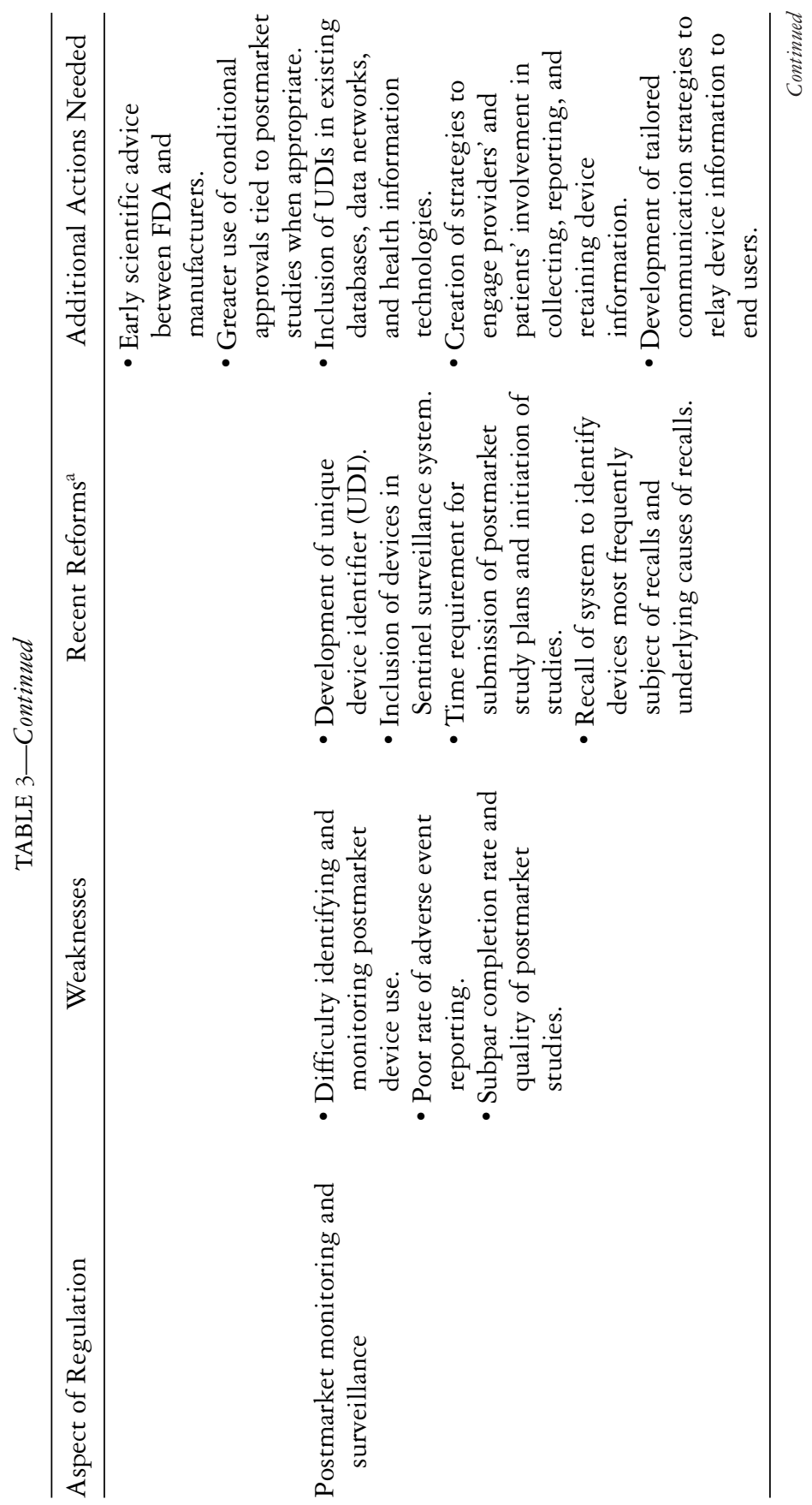




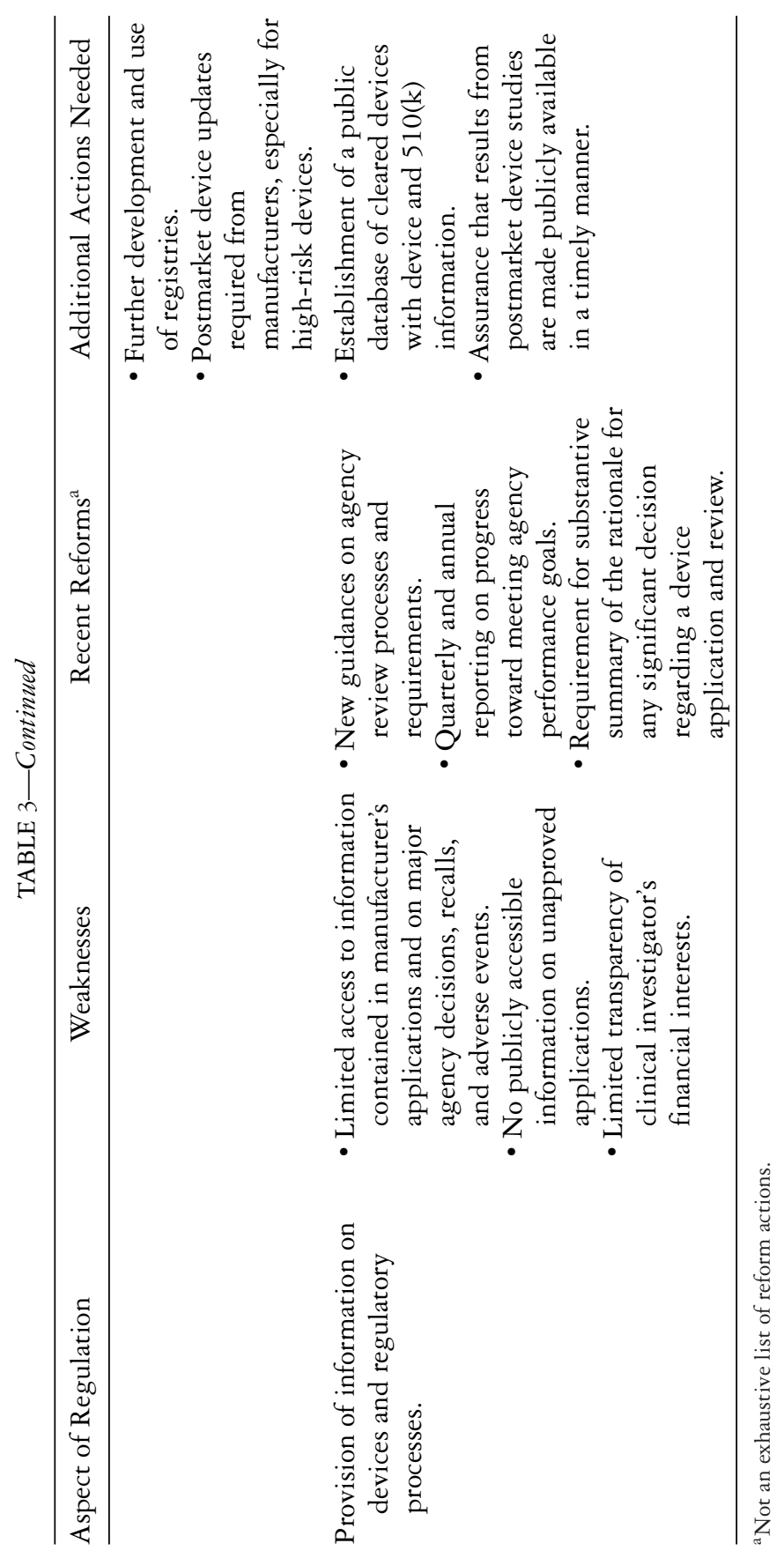




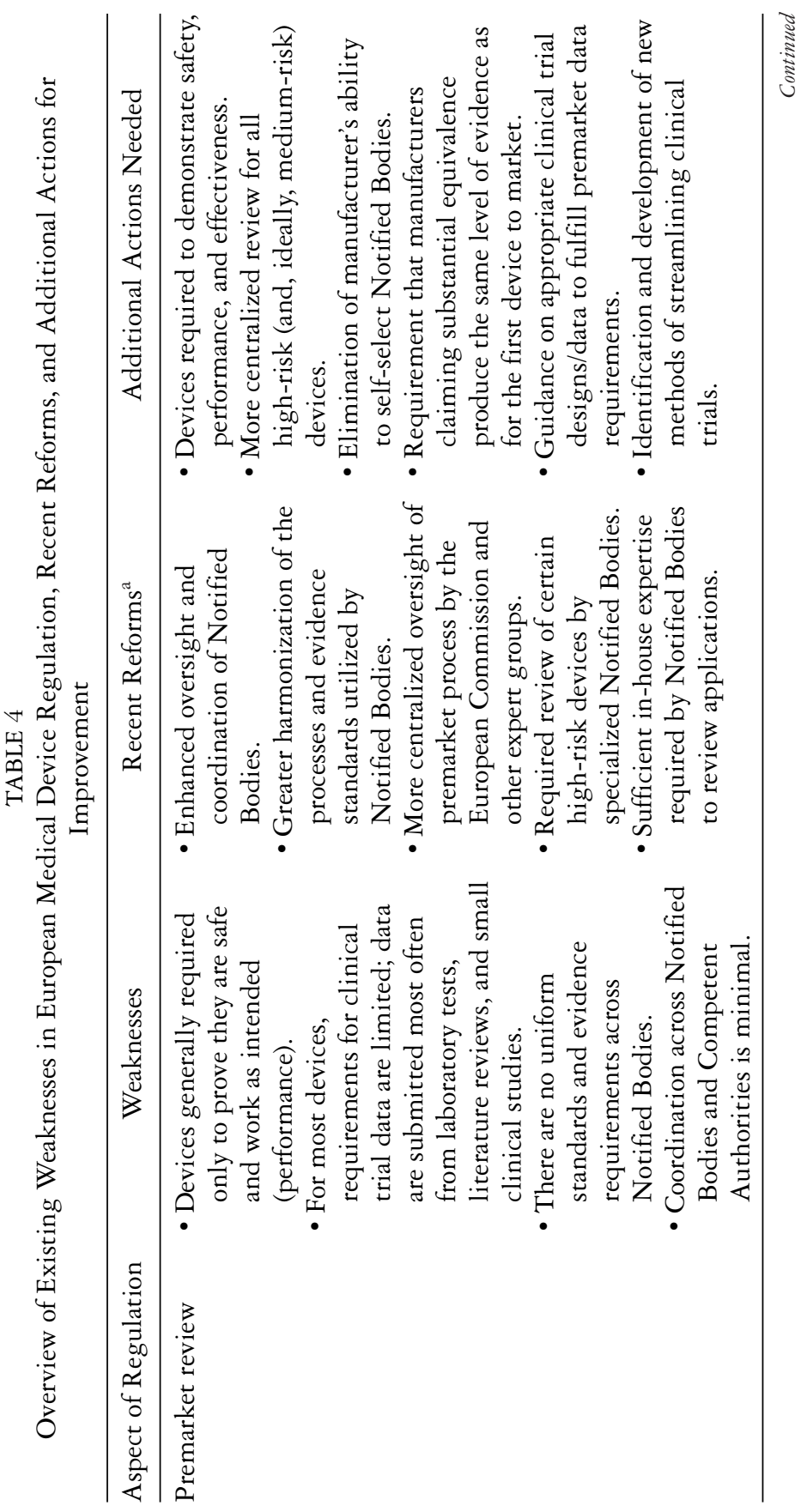




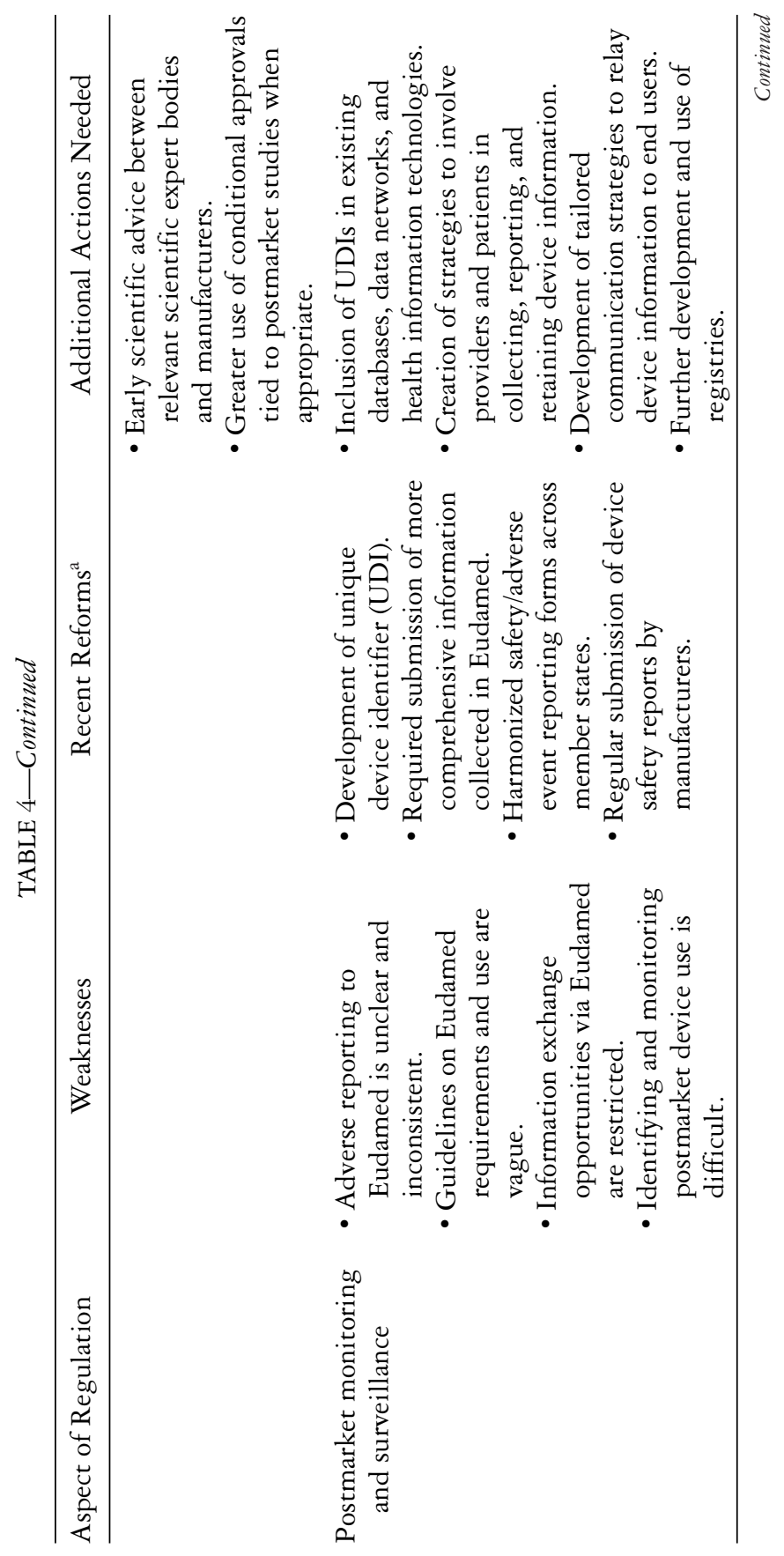




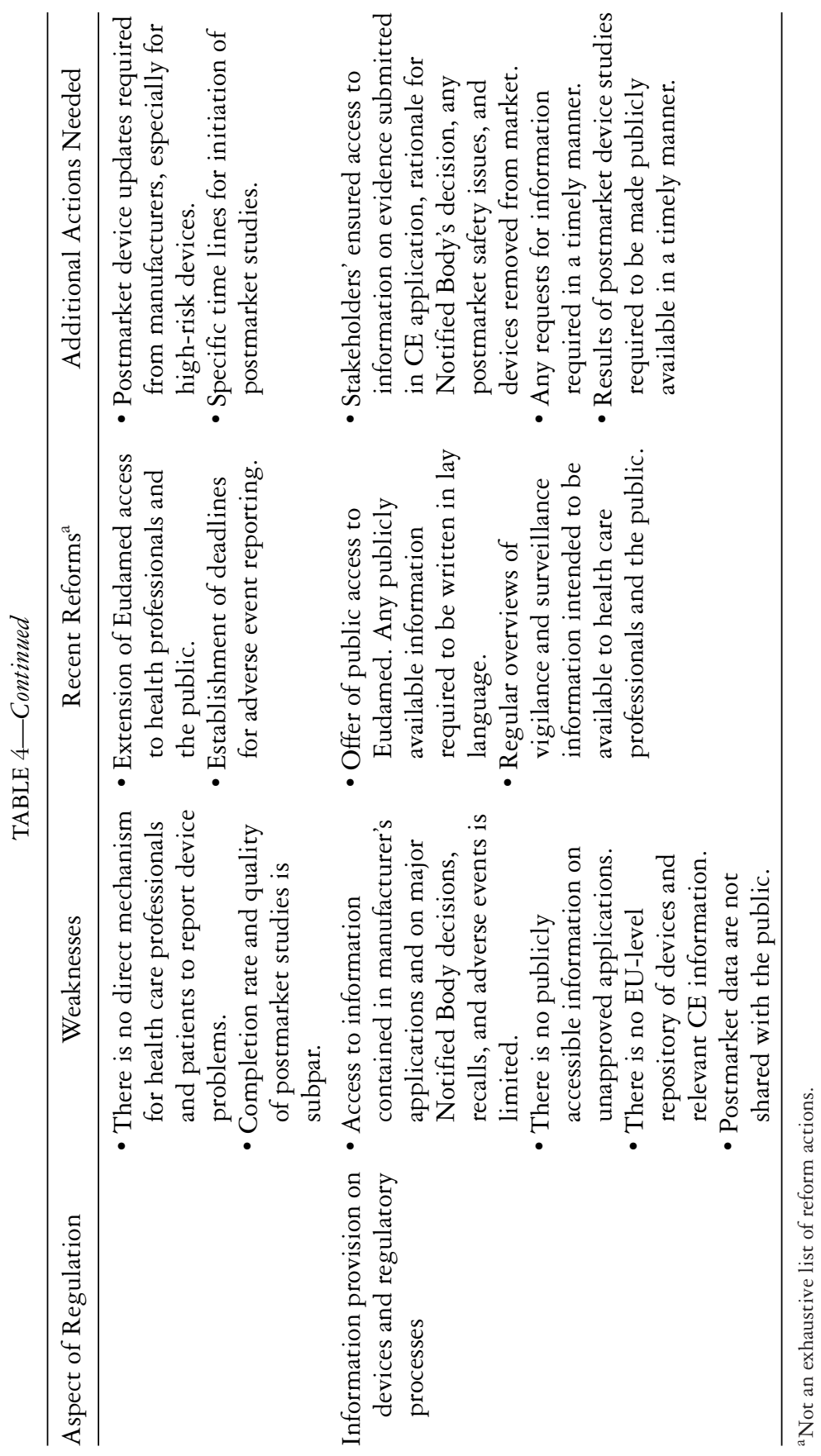


least resistance in which devices are approved more rapidly and on the basis of less evidence.

In line with more robust evidence requirements, European device reforms ideally would extend beyond the Notified Bodies' enhanced oversight. In particular, the reforms should contain a centralized review and approval process for high-risk (and, ideally, medium-risk) devices, with the approval of all other devices going through the Notified Bodies as usual. It is encouraging that the latest reform proposals are moving in this direction by requiring specialized Notified Bodies to review certain high-risk devices. Moreover, it will be important to ensure that all Notified Bodies have enough in-house expertise to review an increasingly diverse range of devices. The new requirements for standardized processes, evidence requirements, and fees for Notified Bodies should protect against manufacturers "cherry-picking" the easiest and fastest option. It may be prudent, however, to eliminate manufacturers' ability to select the Notified Body to which they submit their applications.

In the United States, along with completing the reclassification process for devices on the market before 1976, the FDA should apply more stringent standards for acceptable predicates. Hines and colleagues ${ }^{48}$ discussed several issues with the existing use of predicates, including the permissive interpretation of intended use, disparate technological characteristics between the new device and predicate, and "predicate creep" (over time, a new device can differ quite a bit from that of the original predicate). The agency has started to better clarify the use of predicates, which should help address some of these issues. Periodic audits of $510(\mathrm{k})$ applications and decisions may also help improve their adequacy, accuracy, and consistency.

Both jurisdictions could also encourage manufacturers to conduct premarket studies. The current systems tend to reward "fast followers" to market that can take advantage of existing evidence from already marketed products. If eliminating the use of predicates in premarket approval is not possible, fast followers could be required to generate the same clinical evidence as for other devices already on the market, unless there is compelling evidence of their comparable manufacture. Under such an approach, the first to market would set the evidence standard. This not only would reward those manufacturers first to market by protecting against other manufacturers benefiting from their investment in clinical studies but also would ensure that each new device is supported with evidence regarding its effectiveness, safety, and quality. 
Other actions to support the conduct of clinical trials and submission of quality clinical data are guidance on appropriate clinical trial designs to fulfill premarket data requirements, new methods of streamlining clinical trials, and early scientific advice exchanged between the FDA and manufacturers.

When the evidence is insufficient at the time of approval, market access should be conditioned on rigorous prospective postmarketing studies to substantiate effectiveness and safety in real-world settings. Conditional approval would be one way to support innovation without burdensome overregulation while ensuring patients' safety. Given manufacturers' poor record of completing such studies, US and European regulators should monitor studies more closely and take enforcement actions when they are delayed. In addition, comprehensive information on completed postapproval studies, including trial results, should be made easily accessible online. This would strengthen regulatory decisions and support "downstream" regulation by providing more robust evidence from which to inform pricing and reimbursement decisions. Ongoing investments in postmarket data networks, such as Sentinel, electronic medical records, and UDI, may also facilitate greater use of conditional approvals through better postmarket data collection and analysis.

\section{Postmarket Surveillance}

Ensuring proactive, not passive, postmarketing systems is just as important as strengthening premarket authorization. While reforms on the use of UDIs are a good step toward enabling the tracking and identification of devices, the true benefit of the UDI system will require its broad adoption and use by manufacturers, payers, providers, patients, and other stakeholders involved throughout the life cycle of medical devices. Accordingly, we need strategies to facilitate the awareness, adoption, and implementation of the UDI system. Such efforts should focus on including UDIs in inventory logs, electronic health records, and claims data and linking different postmarket databases, such as the GUDID and adverse event reporting repositories. Moreover, providers and patients should be engaged early to report, receive, and retain device information as well as to tailor strategies for communicating information (eg, smart phone applications that can link the identifier to the UDI database) to different end users. 
The UDI should be included in and facilitate the use of registries. Registries, which collect data on large numbers of patients using observational methods, may be a good way to monitor the use of devices in clinical practice and evaluate their long-term safety and performance. Both Europe and the United States have used registries to collect and evaluate postmarket data, especially in cardiology and orthopedics. For example, the National Joint Registries currently operate in the United Kingdom, Germany, and Italy, collecting information on hip, knee, and/or ankle replacement operations to monitor the devices' performance. In the United States, the Kaiser Permanente Cardiac Device Registry tracks and monitors pacemakers and ICDs, with data on more than 22,000 ICD pulse generators, 52,000 pacemakers, and 90,000 leads. The registry allows the analysis of implant statistics, including complications, failures, replacements, usage, and costs.

Registries have been instrumental in identifying potential problems with a device or its use in practice. ${ }^{49}$ A recent analysis of the United Kingdom and French registries for transcatheter aortic valve implantation (TAVI) found that $25 \%$ and $20 \%$ of patients, respectively, were being treated transapically, which far exceeds what is justified by the clinical evidence and outside use approved by the FDA. ${ }^{50}$ The aforementioned SCAAR study on DES is another example. ${ }^{38}$

Nonetheless, the use of registries needs to be improved. Because there is currently no consensus regarding which devices registries should include, we need criteria for when a device should be captured in a registry as a condition of approval and which devices might produce the most public health benefit from inclusion in registries. Ideally, this would also involve regulators working with stakeholders to establish basic standards for registries regarding methods, data quality, and transparency. Some of the main challenges with registries are adequately accounting for potential bias and the variability in the treatments, population, and settings captured, as well as the continuous development of devices. Creating a registries forum or consortium would be one way to bring the relevant parties together to share best practices and develop new methodologies for registries' data collection and analysis. New registries should also be linked to routinely collected health data, national mortality statistics, claims data, electronic health records, and other possible sources of relevant information. The implementation of UDIs should, in principle, increase the linkage of data. 
Finally, given the continuous evolution of devices, regulators should require periodic update reports from manufacturers, especially for Class III devices. This could encompass any information relevant to the device's benefits and risks, including new study results or scientific assessments of the device's risk-benefit ratio and estimates of the population exposed to the device. European regulators might stipulate a time frame for beginning postmarket device studies, similar to that of the FDA. Both jurisdictions should ensure that the results are publicly available in a timely manner.

\section{Transparency of Processes}

Recent reforms, especially in Europe, have concentrated on improving transparency in device regulation. One particular focus of the proposals is improving the public's and health care professionals' access to information. These stakeholders must have access to comprehensive information on the data submitted in the application (with due regard to commercial confidentiality when justified), the rationale for the Notified Body's decision, any postmarket safety issues or defects, and devices that have been removed from the market. The European Public Assessment Reports (EPARs) used by the EMA may provide a model for communicating this type of summary. In addition, any request for information about a device not available publicly (from health care professionals, the public, Competent Authorities, the commission, etc.) should be addressed without delay.

In the United States, a public database of cleared devices would further aid transparency. The database could contain information about the device, a $510(\mathrm{k})$ summary, predicates used, and any other details pertinent to the clearance decision.

\section{Concluding Remarks}

Regulatory systems for medical devices have an important role in supporting market access to technological innovations while duly protecting the public's health. In order to meet this aim, robust premarket assessment and postmarket vigilance are required. Both the United States and Europe have recently introduced or are in the process of establishing reforms to meet this end. Such initiatives should be implemented in a 
timely manner, though additional actions will be required to enhance the reforms' effectiveness. More research is needed to assess the ongoing performance of regulatory approaches for devices.

\section{References}

1. Kruger K, Kruger M. The medical device sector. In: Burns LR, ed. The Business of Healthcare Innovation. Cambridge: Cambridge University Press; 2012:376-445.

2. US Food and Drug Administration. A delicate balance: FDA and the reform of the medical device approval process. Testimony before Special Committee on Agency, US Senate, 112th Cong., 1st sess., April 13, 2011. www.fda.gov/NewsEvents/Testimony/ ucm250709.htm. Accessed December 5, 2011.

3. Institute of Medicine. Medical Devices and the Public's Health. The FDA 510(k) Clearance Process at 35 Years. Washington, DC: National Academies Press; 2011. http://www.iom.edu/Reports/ 2011/Medical-Devices-and-the-Publics-Health-The-FDA-510kClearance-Process-at-35-Years.aspx. Accessed November 20, 2011.

4. Miller R. Debate on 510(k)s and recalls reaches Capitol Hill. Medscape. February 18, 2011. www.medscape.com/viewarticle/737721. Accessed October 15, 2011.

5. Gottlieb S. How the FDA could cost you your life. Wall Street Journal. October 3, 2011: A17. online.wsj.com/news/articles/ SB10001424052970204831304576597200095602270. Accessed January 9, 2012.

6. Pollack A. Medical treatment, out of reach. New York Times. February 9, 2011. www.nytimes.com/2011/02/10/business/10device. html?pagewanted=all\&_r=0. Accessed October 28, 2013.

7. Freemantle N. Evaluating and regulating the device industry. BMJ. 2011;342:d2839.

8. European Commission. Roadmap 2012: Proposal for a Directive of the European Parliament and of the Council Concerning Medical Devices and Repealing Directives 90/385/EEC and 93/42/EEC. Brussels: European Commission; 2011. ec.europa.eu/governance/impact/ planned_ia/docs/2008_sanco_081_proposal_medical_devices_en. pdf. Accessed October 10, 2011.

9. Heneghan C. The saga of Poly Implant Prothèse breast implants. BMJ. 2011;344:e306.

10. Meier B. FDA to tighten regulation of all-metal hip implants. New York Times. January 16, 2013. http://www.nytimes. 
com/2013/01/17/business/fda-to-tighten-regulation-of-all-metalhip-implants.html. Accessed August 5, 2013.

11. US Food and Drug Administration. Unsafe and ineffective devices approved in the EU that were not approved in the US. Silver Spring, MD: US Food and Drug Administration; 2012. http://www.elsevierbi.com/ /media/Supporting\%20Documents/ The\%20Gray\%20Sheet/38/20/FDA_EU_Devices_Report.pdf. Accessed October 30, 2013.

12. Kramer DB, Xu S, Kesselheim AS. Regulation of medical devices in the United States and European Union. $N$ Engl $J$ M. 2012;366(9):848-855.

13. Basu S, Hassenplug JC. Patient access to medical devices-a comparison of U.S. and European review processes. $N$ Engl $J$ M. 2012;367:485-488.

14. US Government Accountability Office. Classes of Devices. Washington, DC: US Government Printing Office. Publication No. 21 USC 360c(a); 2006 ed.

15. US Food and Drug Administration. Device advice: premarket notification. Rockville, MD: Food and Drug Administration; 2000. http://www.fda.gov/medicaldevices/deviceregulationandguidance/ howtomarketyourdevice/premarketsubmissions/ premarketnotification $510 \mathrm{k} /$ default.htm. Accessed October 31, 2013.

16. US Food and Drug Administration. Modifications to devices subject to Premarket Approval (PMA) - the PMA supplement decision-making process. Accessed December 11, 2008. http:// www.fda.gov/downloads/MedicalDevices/DeviceRegulation andGuidance/GuidanceDocuments/ucm089360. Accessed April 3, 2013.

17. Code of Federal Regulations, 21CFR814.82, April 1, 2012.

18. Fraser AG, Krucoff MW, Brindis R, Komajda M, Smith SC Jr. International collaboration needed on device clinical standards. BMJ. 2011;342:d2952.

19. Cohen D, Billingsley M. Europeans are left to their own devices. BMJ. 2011;342:d2748.

20. Thompson M, Heneghan C, Billingsley M, Cohen D. Medical device recalls and transparency in the UK. BMJ. 2011;342:d2973.

21. Sweet BV, Schwemm AK, Parsons DM. Review of the processes for FDA oversight of drugs, medical devices, and combination products. J Manage Care Pharm. 2011;17:40-50.

22. US Government Accountability Office. Medical devices: FDA should take steps to ensure that high-risk device types are approved through the most stringent premarket review process. 
Washington, DC: US Government Printing Office; 2009. Publication No. GAO-09-190. www.gao.gov/new.items/d09190.pdf. Accessed October 31, 2013.

23. US Government Accountability Office. Medicare durable medical equipment: Class III devices do not warrant a distinct annual payment update. Washington, DC: US Government Printing Office; 2006. Publication No. GAO-06-02. www.gao.gov/new. items/d0662.pdf. Accessed October 30, 2013.

24. Ardaugh BM, Graves SE, Redberg RF. The 510(k) ancestry of a metal-on-metal hip implant. N Engl J M. 2013;368:97-100.

25. Zuckerman DM, Brown P, Nissen SE. Medical device recalls and the FDA approval process. Arch Intern Med. 2011; 171(11):10061011.

26. US Food and Drug Administration. Understanding Barriers to Medical Device Quality. Silver Spring, MD: US Food and Drug Administration; 2011.

27. US Food and Drug Administration. Guidance for industry and Food and Drug Administration staff-factors to consider when making benefit-risk determinations in medical device premarket approvals and de novo classifications. Silver Spring, MD: US Food and Drug Administration; 2012. www.fda.gov/medicaldevices/ deviceregulationandguidance/guidancedocuments/ucm267829. htm. Accessed November 1, 2013.

28. Dhruva SS, Bero LA, Redbury RF. Strength of study evidence examined by the FDA in premarket approval of cardiovascular devices. JAMA. 2009;302:2679-2685.

29. Chen CE, Dhruva SS, Bero LA, Redberg RF. Inclusion of training patients in US Food and Drug Administration premarket approval cardiovascular device studies. Arch Intern Med. 2011;171:534-539.

30. Chen CE, Dhruva SS, Redberg RF. Inclusion of comparative effectiveness data in high-risk cardiovascular device studies at the time of premarket approval. JAMA. 2012;308(17):1740-1742.

31. US Food and Drug Administration. Guidance for industryproviding clinical evidence of effectiveness for human drug and biological products. Rockville, MD: US Food and Drug Administration; 1998. www.fda.gov/downloads/Drugs/GuidanceCompliance RegulatoryInformation/Guidance/ucm078749.pdf. Accessed October 30, 2013.

32. Miller R. Studies show cardiovascular devices often earn FDA approval without high-quality clinical data. Medscape. December 30, 2009. www.medscape.com/viewarticle/790524? $\mathrm{t}=1$. Accessed October 30, 2013.

33. Di Mario C, James S, Dudek D, Sabate M, Degertekin M. Commentary: The risk of over-regulation. BMJ. 2011;342:d3021. 
34. Lenzer J, Brownlee S. Why the FDA can't protect the public. BMJ. 2010;341:c4753.

35. Office of the Inspector General. Adverse event reporting for medical devices. Boston, Mass: Inspector General, Department of Health and Human Services; 2009. doi: OEI-01-08-00110. oig.hhs.gov/oei/reports/oei-01-08-00110.pdf. Accessed November 11, 2011.

36. Malenka DJ, Kaplan AV, Sharp SM, Wennberg JE. Post-marketing surveillance of medical devices using Medicare claims. Health Aff. 2005;24(4):928-937.

37. Cheng M. Medical Device Regulations: Global Overview and Guiding Principles. Geneva, Switzerland: World Health Organization; 2003.

38. Lagerqvist B, James SK, Stenestrand U, et al. Long-term outcomes with drug-eluting stents versus bare-metal stents in Sweden. $N$ Engl J M. 2009;356:1009-1019.

39. Sarno G, Lagerqvist B, Frober O, et al. Lower risk of stent thrombosis and restenosis with unrestricted use of "new-generation" drug-eluting stents: a report from the nationwide Swedish Coronary Angiography and Angioplasty Registry (SCARR). Eur Heart J. 2012. doi:10.1093/eurheart/ehr479.

40. US Food and Drug Administration. Guidance for clinical investigators, industry, and FDA staff-financial disclosure by clinical investigators. Silver Spring, MD: US Food and Drug Administration; 2013. www.fda.gov/downloads/RegulatoryInformation/ Guidances/UCM341008.pdf. Accessed October 30, 2013.

41. Federal Register. Food and Drug Administration Safety and Innovation Act (S.3187). Washington, DC: US Government Printing Office; 2012. http://www.gpo.gov/fdsys/pkg/PLAW112publ144/pdf/PLAW-112publ144.pdf. Accessed April 5, 2013.

42. European Commission. Proposal for a regulation of the European Parliament and of the Council on Medical Devices, and amending directive 2001/83/EC, regulation (EC) No 178/2002 and regulation (EC) No 1223/2009. Brussels: European Commission; 2012. ec.europa.eu/health/medical-devices/files/revision_docs/ proposal_2012_542_en.pdf. Accessed on March 10, 2013.

43. European Parliament. Draft report on the proposal for a regulation of the European Parliament and of the Council on Medical Devices, and amending directive 2001/83/EC, regulation (EC) No 178/2002 and regulation (EC) No 1223/2009. Brussels: European Commission, Committee on the Environment, Public Health and Food Safety; 2013. http://www.europarl.europa.eu/sides/getDoc.do?pubRef=$\% 2 \mathrm{~F} \% 2 \mathrm{FEP} \% 2 \mathrm{~F} \% 2 \mathrm{FNONSGML} \% 2 \mathrm{BCOMPARL} \% 2 \mathrm{BPE}-$ 
$507.972 \% 2 \mathrm{~B} 02 \% 2 \mathrm{BDOC} \% 2 \mathrm{BPDF} \% 2 \mathrm{BV} 0 \% 2 \mathrm{~F} \% 2 \mathrm{FEN}$.

Accessed November 1, 2013.

44. Cohen D. Devices and desires: industry fights toughening of medical device regulation in Europe. BMJ. 2013;347:f6204. doi: 10.1136/bmj.f6204.

45. Ladin Z, Imhoff M. FDA review patterns of de novo submissions. Boston: Boston MedTech Advisors; 2010. bmtadvisors.com/docs/2010_06_10_FDA\%20Review\%20Patterns\%20of $\% 20 \mathrm{De} \% 20$ Novo\%20Submissions_Final.pdf. Accessed October $25,2013$.

46. US Food and Drug Administration. Analysis of premarket review times under the $510(\mathrm{k})$ program. Silver Spring, MD: US Food and Drug Administration; 2011. www.fda.gov/aboutfda/ centersoffices/officeofmedicalproductsandtobacco/cdrh/cdrhreports/ ucm 263385.htm. Accessed October 25, 2013.

47. US Food and Drug Administration. Improvements in device review-results of CDRH's plan of action for premarket review of devices. Silver Spring, MD: US Food and Drug Administration; 2012. www.fda.gov/downloads/AboutFDA/ ReportsManualsForms/Reports/UCM329702.pdf. Accessed November 2, 2013.

48. Hines JZ, Lurie P, Yu E, Wolfe S. Left to their own devices: breakdowns in United States medical device premarket review. PLoS Med. 2010;7(7):e1000280.

49. James S, Daubert JC, Van de Werf F. Use of registries to investigate the past and develop the future. BMJ. 2011;342:d2826.

50. Van Brabandt H, Neyt M, Hulstaert F. Transcatheter aortic value implantation (TAVI): risky and costly. BMJ. 2012;345:e4710.

Acknowledgments: We would like to thank the Commonwealth Fund for supporting this research. LSE received an unrestricted grant to investigate international health policy issues. The views presented in this article are those of the authors and do not necessarily reflect the Fund's position. We also are indebted to Professor Robert Burns at the University of Pennsylvania for his valuable input into an earlier draft of the article.

Disclosure: Authors both have appointments at LSE, which received an unrestricted grant from Eucomed (a medical devices industry association). 